\title{
Towards Exploratory Hypothesis Testing and Analysis
}

\author{
Guimei Liu ${ }^{\# 1}$, Mengling Feng ${ }^{* 2}$, Yue Wang ${ }^{\# 3}$, Limsoon Wong ${ }^{* 4}$, See-Kiong Ng ${ }^{* 5}$, \\ Tzia Liang Mah ${ }^{* 6}$, Edmund Jon Deoon Lee ${ }^{* * 7}$ \\ \# Department of Computer Science, National University of Singapore, Singapore \\ $\left\{{ }^{1}\right.$ liugm, ${ }^{4}$ wongls $\} @$ comp.nus.edu.sg \\ * Data Mining Department, Institute for Infocomm Research, Singapore

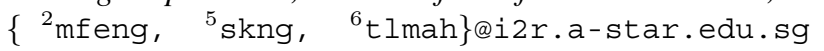 \\ \#\# Graduate School for Integrative Science and Engineering, National University of Singapore, Singapore \\ ${ }^{3}$ wangyue@nus.edu.sg \\ ${ }^{* *}$ Pharmacology Department, National University of Singapore, Singapore \\ ${ }^{7}$ edlee@nus.edu.sg
}

\begin{abstract}
Hypothesis testing is a well-established tool for scientific discovery. Conventional hypothesis testing is carried out in a hypothesis-driven manner. A scientist must first formulate a hypothesis based on his/her knowledge and experience, and then devise a variety of experiments to test it. Given the rapid growth of data, it has become virtually impossible for a person to manually inspect all the data to find all the interesting hypotheses for testing. In this paper, we propose and develop a data-driven system for automatic hypothesis testing and analysis. We define a hypothesis as a comparison between two or more sub-populations. We find sub-populations for comparison using frequent pattern mining techniques and then pair them up for statistical testing. We also generate additional information for further analysis of the hypotheses that are deemed significant. We conducted a set of experiments to show the efficiency of the proposed algorithms, and the usefulness of the generated hypotheses. The results show that our system can help users (1) identify significant hypotheses; (2) isolate the reasons behind significant hypotheses; and (3) find confounding factors that form Simpson's Paradoxes with discovered significant hypotheses.
\end{abstract}

\section{INTRODUCTION}

Hypothesis testing is a well-established tool for scientific discovery. It enables scientists to distinguish results that represent systematic effects in the data from those that are due to random chance. Hypothesis testing involves a comparison of two or more sub-populations. One example hypothesis is "Smokers are more vulnerable to the H1N1 flu than nonsmokers". To test this hypothesis, we need to compare the occurrence of H1N1 flu infection between two sub-populations; in this case, smokers and non-smokers. The outcome of hypothesis testing can help people make decisions. For example, knowing which group of people are more vulnerable to a certain type of flu, doctors can vaccinate this group of people first to prevent the spread of the flu.

Conventional hypothesis testing is usually carried out in a hypothesis-driven manner. A scientist must first formulate a hypothesis based on his/her knowledge and experience, and then devise a variety of experiments to test it. This presents a possible catch-22 situation, for it is often the case that people want to find something from their data, but do not know what to find. Even if a person has much domain knowledge and ample experience, the data may still contain something useful that he/she is not aware of. For example, even an experienced doctor may not know all the risk factors of a complex disease.

With the rapid development and wide usage of computer technologies, more and more data have been accumulated and stored in digital format. These data provide rich sources for making new discoveries. However, the sheer volume of the data available nowadays makes it impossible for people to inspect all the data manually. As a result, lots of useful knowledge may go undiscovered. This calls for the need of developing a system for automatic hypothesis testing in a datadriven manner.

Data mining is an important tool to transform data into knowledge in a data-driven manner. Data mining does not start from a pre-conception or a specific question like hypothesis testing. Instead, it aims to automatically extract useful information from large volumes of data via exploratory search, making it highly applicable for automatic knowledge discovery. In this paper, we develop a system for exploratory hypothesis testing and analysis by building on and extending existing data mining techniques. Given a dataset, we formulate and test tentative hypotheses based on the attributes in the dataset, the nature of the attributes and the statistics of the data. The space of all the possible hypotheses can be very large. We employ techniques developed for frequent pattern mining [2] to efficiently explore the space of tentative hypotheses.

In many cases, it is not sufficient to just know whether a hypothesis is statistically significant. It is more interesting and important to know the reasons behind significant hypotheses. Therefore, we further analyze the hypotheses that are statistically significant and identify the factors that contribute to the difference. Another reason for the need of further analysis of significant hypotheses is that some of the significant hypotheses generated by exploratory search may be spurious since the exploration is not guided by domain 
knowledge. We examine whether there are any confounding factors that may lead to spurious hypotheses.

The rest of the paper is organized as follows. Section II formally defines the exploratory hypothesis testing and analysis problem. In Section III, we develop efficient algorithms for automatic hypothesis testing and analysis by using and extending well-established frequent pattern mining techniques. Experiment results are reported in Section IV. Section V describes related work. Finally, in Section VI, we conclude the paper with some closing discussions.

\section{PRoBlem DEFINITION}

Hypothesis testing is a test of significance on a difference. A difference is statistically significant if it is unlikely to have occurred by chance. Hypothesis testing can be conducted on $n$ sub-populations, where $n \geq 1$. Hypothesis testing involving only one sub-population compares the statistics of one subpopulation with the parameters of the general population. However, the parameters of the general population are often unknown. The results of hypothesis testing involving more than two sub-populations are usually hard to interpret. It is not easy for users to tell which sub-population contributes the most to the difference. In the end, users still need to resort to pairwise comparisons to get a clear picture. Hence, we focus on the case when $n=2$ in this paper.

The hypothesis testing process consists of four main steps:

1) State the relevant null and alternative hypotheses. The null hypothesis is always "There is no difference." and the alternative hypothesis is "There is difference".

2) Choose a proper statistical test based on the type and distribution of the attribute that the sub-populations are compared on. An overview of the statistical tests and when should they be used can be found at [17].

3) Calculate the p-value using the chosen test. The p-value is the probability of obtaining a statistic at least as extreme as the one that was actually observed, given the null hypothesis is true. The lower the p-value, the less likely that the observed difference is due to random chance, thus the more statistically significant the difference is.

4) Decide whether to reject or accept the null hypothesis based on the p-value. Conventionally, a p-value of 0.05 is generally recognized as low enough to reject the null hypothesis and accept the alternative hypothesis.

In the rest of this section, we describe how to automate the testing process to test all the possible hypotheses in a given dataset. We define two tasks, exploratory hypothesis testing and hypothesis analysis, in the situation when the target attribute is categorical. The target attribute is the attribute on which the significance of the difference is tested. It is straightforward to generalize the definitions to the situation when the target attribute is continuous.

\section{A. Hypothesis formulation}

We use the example dataset shown in Table I to illustrate how to formulate hypotheses. Each row in the dataset is the medical record of a patient. The last column is the response of the patients to a certain treatment. One example hypothesis is "Treatment A is more effective than treatment B". The two sub-populations under comparison are "patients undergone treatment A" and "patients undergone treatment B", and they are compared on attribute "Response". There are two types of attributes in a hypothesis. One type of attributes, such as "Treatment", are used to define sub-populations, and we call them grouping attributes. A grouping attribute cannot be continuous. The other type of attributes, such as "Response", are the attribute on which the significance of the difference is tested, and we call them target attributes.

TABLE I

AN EXAMPLE DATASET

\begin{tabular}{|c|c|c|c|c|c|c|c|}
\hline PID & Race & Gender & Age & Smoke & Stage & Treatment & Response \\
\hline 1 & Caucasian & M & 45 & Yes & 1 & A & positive \\
2 & Asian & M & 40 & No & 1 & A & positive \\
3 & African & F & 50 & Yes & 2 & B & negative \\
$\vdots$ & $\vdots$ & $\vdots$ & $\vdots$ & $\vdots$ & $\vdots$ & $\vdots$ & $\vdots$ \\
N & Caucasian & M & 60 & No & 2 & B & negative \\
\hline
\end{tabular}

Given a dataset, we ask domain users to specify a target attribute as the objective of the exploratory hypothesis testing. If the target attribute is a categorical attribute, we further ask domain users to choose a value that is the most interesting to them, and we call this value target attribute value. We also ask domain users to specify the grouping attributes if possible (if not, we simply use all the categorical attributes in the given dataset as grouping attributes). This should be easy for domain users since they usually have some rough ideas on which attributes are of interest for comparison and which attributes can be used for grouping.

A sub-population is defined by a set of attribute-value pairs. We call an attribute-value pair an item, and a set of items $a$ pattern.

Definition 1 (Pattern): A pattern is a set of attribute-value pairs (items), denoted as $P=\left\{A_{1}=v_{1}, A_{2}=v_{2}, \cdots, A_{k}=\right.$ $\left.v_{k}\right\}$, where $A_{i}$ is an attribute $(1 \leq i \leq k), v_{i}$ is a value taken by attribute $A_{i}$, and $A_{i} \neq A_{j}$ if $i \neq j$.

If an attribute-value pair $A=v$ appears in a pattern $P$, we say $P$ contains attribute $A$. Each pattern defines a subpopulation. For example, pattern $\{$ EthnicGroup=Caucasian, Gender= Male $\}$ defines the sub-population consisting of Caucasian male patients. In the rest of this paper, when we say pattern $P$, we refer to the pattern itself; when we say subpopulation $P$, we refer to the sub-population defined by $P$. The support of a pattern $P$, is defined as the number of records containing $P$, denoted as $\sup (P)$. The support of pattern $P$ is equivalent to the size of sub-population $P$.

Given two patterns $P$ and $P^{\prime}$, if every item in $P$ is also in $P^{\prime}$, then $P$ is called a sub-pattern of $P^{\prime}$, denoted as $P \subseteq P^{\prime}$, and $P^{\prime}$ is called a super-pattern of $P$, denoted as $P^{\prime} \supseteq P$. If $P$ is a sub-pattern of $P^{\prime}$ and $P$ has one less item than $P^{\prime}$, then we call $P$ an immediate sub-pattern of $P^{\prime}$, and $P^{\prime}$ an immediate super-pattern of $P$.

In hypothesis testing, users usually study one factor at a time. Hence, in this work, we require that the defin- 
ing patterns of two sub-populations under comparison differ by one and only one item. For example, comparing subgroup $\{$ EthnicGroup $=$ Caucasian, Gender $=$ Male $\}$ with subgroup $\{$ EthnicGroup $=$ Caucasian, Gender $=$ Female $\}$ is accept able, while comparing subgroup $\{$ EthnicGroup=Caucasian, Gender $=$ Male $\}$ with subgroup $\{$ EthnicGroup=Asian, Gender $=$ Female $\}$ is less intuitive because even if the difference between the two sub-populations is statistically significant, it is hard for users to conjecture which attribute contributes to the difference. Now we formally define tentative hypotheses.

Definition 2 (Tentative hypothesis): Let $A_{\text {target }}$ be a categorical target attribute, $v_{\text {target }}$ be the target attribute value, $P_{1}$ and $P_{2}$ be two patterns that contain the same set of attributes and differ by one and only one item, denoted as $P_{1}=P \cup\left\{A_{\text {diff }}=v_{1}\right\}, P_{2}=P \cup\left\{A_{\text {diff }}=v_{2}\right\}$. The tentative hypothesis on the two sub-populations defined by $P_{1}$ and $P_{2}$ is represented as $H=\left\langle P, A_{\text {diff }}=v_{1} \mid v_{2}, A_{\text {target }}, v_{\text {target }}\right\rangle$. Pattern $P$ is called the context of $H$, attribute $A_{\text {diff } f}$ is called the comparing attribute of $H$, and $P_{1}$ and $P_{2}$ are called the two sub-populations of $H$. The null hypothesis is $p_{1}=p_{2}$, and the alternative hypothesis is $p_{1} \neq p_{2}$, where $p_{i}$ is the proportion of $v_{\text {target }}$ in $P_{i}$, i.e., $p_{i}=\frac{\sup \left(P_{i} \cup\left\{A_{\text {target }}=v_{\text {target }}\right\}\right)}{\sup \left(P_{i}\right)}, i=1,2$.

Based on the definition, hypothesis "Treatment $A$ is more effective than treatment B" can be represented as $\langle\{\}$, Treatment $=A| B$, Response, positive $\rangle$.

\section{B. Choosing a proper test and calculating the p-value}

The selection of a proper statistical test depends on the type and distribution of the target attribute. An overview of the statistical tests and when should they be used can be found at [17]. Most of these tests can be integrated into our system seamlessly. Given a statistical test, we usually need to scan the dataset and collect some statistics for the calculation of the p-value.

TABLE II

CALCUlating THE P-VALUE OF HYPOTHESIS “TREaTMENT A IS MORE EFFECTIVE THAN TREATMENT B"

\begin{tabular}{|l|l|c|}
\hline Patterns & $\sup$ & $p_{i}$ \\
\hline Treatment=A & $1000\left(n_{1}\right)$ & $p_{1}=89 \%$ \\
Treatment=A, Response=positive & 890 & \\
\hline Treatment=B & $1000\left(n_{2}\right)$ & $p_{2}=83 \%$ \\
Treatment=B, Response=positive & 830 & \\
\hline
\end{tabular}

(a) Statistics needed for calculating p-value using $\chi^{2}$-test

\begin{tabular}{|c|c|c|}
\hline Hypothesis $H$ & $\chi^{2}$-score & p-value \\
\hline$\langle\{\}$, Treatment $=A| B$, Response, positive $\rangle$ & 14.95 & 0.0001 \\
\hline
\end{tabular}

(b) p-value

For the example hypothesis $H$ : "Treatment A is more effective than treatment B", the two sub-populations are compared on attribute "Response" and it is nominal, hence we choose $\chi^{2}$-test with Yates' correction[21]. The $\chi^{2}$-test is a test for examining the association between two categorical attributes, and it requires four statistics to be collected: the size of the two sub-populations under comparison, denoted as $n_{1}$ and $n_{2}$, and the proportion of the target attribute value in the two subpopulations, denoted as $p_{1}$ and $p_{2}$. The four values of $H$ are shown in Table II (a). Given the four values, the $\chi^{2}$-score is calculated as follows:

$$
\chi_{Y \text { ates }}^{2}=\frac{\left(n_{1}+n_{2}\right)\left(n_{1} n_{2}\left|p_{1}-p_{2}\right|-\left(n_{1}+n_{2}\right) / 2\right)^{2}}{n_{1} n_{2}\left(n_{1} p_{1}+n_{2} p_{2}\right)\left(n_{1}+n_{2}-n_{1} p_{1}-n_{2} p_{2}\right)}
$$

The degree of freedom of this score is 1 . From $\chi^{2}$-score and the degree of freedom, we can get the corresponding p-value by looking up a $\chi^{2}$ distribution table. Table II (b) shows the $\chi^{2}$-score and p-value of $H$.

\section{Deciding the significance of the observed difference}

Conventionally, a p-value of 0.05 is generally recognized as low enough to reject the null hypothesis if one single hypothesis is tested. A p-value of 0.05 means that there is a 0.05 probability that the null hypothesis is true but we are wrongly rejecting it. Here we are testing large numbers of hypotheses simultaneously, which may generate many false positives [4]. We use two methods to control the number of false positives, Bonferroni correction [1] and Benjamini and Hochberg's method [4].

Bonferroni correction [1] is one of the most commonly used approaches for multiple comparisons. It aims at controlling family-wise error rate (FWER) - the probability of making one or more false discoveries among all the hypotheses. The basic idea is that if we test $n$ dependent or independent hypotheses, then one way of maintaining the family-wise error rate is to test each individual hypothesis at a statistical significance level of $1 / n$ times what it would be if only one hypothesis were tested. Bonferroni correction is computationally simple, but it can be very conservative and may inflate the rate of false negatives unnecessarily.

Benjamini and Hochberg's method [4] controls false discovery rate (FDR) - the expected proportion of falsely rejected null hypotheses, which is less stringent than family-wise error rate. Let $H_{1}, H_{2}, \cdots, H_{n}$ be the $n$ hypotheses tested and they are sorted in ascending order of p-value. Their corresponding p-values are $p_{1}, p_{2}, \cdots, p_{n}$. To control FDR at a level of $q$, we get the largest $i$, denoted as $k$, for which $p_{i} \leq \frac{i}{n} q$, and then regard all $H_{i}, i=1,2, \cdots, k$, as statistically significant.

We use the above two methods in our system as follows. We ask users to specify a statistical significance threshold max_pvalue for testing one single hypothesis, and output the hypotheses with $\mathrm{p}$-value $\leq$ max _pvalue. During the hypothesis generation process, we count the total number of tests being performed, denoted as $n$. Bonferroni correction can be easily applied by replacing max_pvalue with max_pvalue/n. We then rank the hypotheses in ascending order of p-values, and apply Benjamini and Hochberg's method.

Statistical significance vs. domain significance. Sometimes a statistically significant result can have little or no domain significance. For example, given large sample sizes, a difference in 5 beats per minutes in pulse rate in a clinical trial involving two drugs can give a statistically significant difference whereas the average difference may hardly bring about a drastic metabolic change between the two groups. The reason being that domain significance mainly depends on the difference between the two means or proportions, 
while statistical significance also depends on standard error. If standard error is small enough, a difference can be statistically significant even if it is not domain significant. Only domain users can decide the level at which a result is regarded as domain significant.

\section{Hypothesis analysis}

In many cases, we are not only interested in knowing whether the difference between two sub-populations is significant, we are more interested in knowing the reasons behind the difference. For example, given the failure rate of one model of a product is significantly higher than that of another model, it is important for engineers to know under what situations the first model is more likely to fail so that they can improve its design accordingly. Table III compares the failure rate of two models of the same product. Model A has a higher failure rate than model $\mathrm{B}$ in general. However, after the two sub-populations are further divided using attribute "time-offailure", we find that model A has comparable failure rate with model B at the time of "in-operation" and "outputting", but has exceptionally high failure rate in the "loading" phase. This information is very useful since it helps engineers narrow down the problem.

TABLE III

AN EXAMPLE INTERESTING ATTRIBUTE "TIME-OF-FAILURE". "loading” is of particular interest because the two models show a very big difference.

\begin{tabular}{|l|c|}
\hline pairs of & $\begin{array}{c}\text { failure } \\
\text { rates }\end{array}$ \\
\hline sub-populations & $4 \%$ \\
model=A & $2 \%$ \\
\hline model=B $=A$, time-of-failure=loading & $6.0 \%$ \\
model=B, time-of-failure=loading & $1.9 \%$ \\
\hline model=A, time-of-failure=in-operation & $2.1 \%$ \\
model=B, time-of-failure=in-operation & $2.1 \%$ \\
\hline model=A, time-of-failure=outputting & $2.0 \%$ \\
model=B, time-of-failure=outputting & $1.9 \%$ \\
\hline
\end{tabular}

TABLE IV

AN EXAMPLE SPURIOUS HYPOTHESIS. Attribute "stage" is a confounding factor.

\begin{tabular}{|c|c|c|c|c|}
\hline $\begin{array}{l}\text { pairs of } \\
\text { sub-populations }\end{array}$ & $\begin{array}{r}\text { res } \\
\text { positive }\end{array}$ & $\begin{array}{l}\text { onse } \\
\text { negative }\end{array}$ & $\begin{array}{c}\text { proportion of } \\
\text { positive response }\end{array}$ & $\mathrm{p}$-value \\
\hline Treatment $=\mathrm{A}$ & 890 & 110 & $89.0 \%$ & 0.0001 \\
\hline Treatment $=\mathrm{B}$ & 830 & 170 & $83.0 \%$ & \\
\hline Treatment $=$ A, Stage $=1$ & 800 & 80 & $90.9 \%$ & 0.0807 \\
\hline Treatment $=B$, Stage $=1$ & 190 & 10 & $95 \%$ & \\
\hline Treatment $=$ A, Stage $=2$ & 90 & 30 & $75 \%$ & 0.2542 \\
\hline Treatment $=B$, Stage $=2$ & 640 & 160 & $80 \%$ & \\
\hline
\end{tabular}

Another reason for the need of further analysis of significant hypotheses is that some of the significant hypotheses generated via exploratory search may be spurious since the search is not guided by domain knowledge. For example, hypothesis $H$ in Table II(b) is actually a spurious hypothesis as shown in Table IV. The original hypothesis $H$ indicates that treatment A is more effective than B. However, after we further divide the two sub-populations of $H$ into smaller subgroups using attribute "stage", we find that treatment B is more effective than treatment A for both patients at stage 1 and patients at stage 2. This phenomenon is called Simpson's Paradox [19], and it is caused by the fact that "stage" has associations with both treatment and response: doctors tend to give treatment A to patients at stage 1 , and treatment B to patients at stage 2 ; patients at stage 1 are easier to cure than patients at stage 2. Attributes that have associations with both the comparing attribute and the target attribute are called confounding factors.

The above examples show that further investigation of hypotheses is often very useful, and it can be conducted by dividing the two sub-populations under comparison into finer subgroups, and then inspecting whether unexpected result is observed in pairs of the finer subgroups.

\section{Difference lift of items and attributes}

To divide the two sub-populations of a hypothesis $H$ into smaller subgroups, we can add more items into the context $P$ of $H$. A simple way to measure the impact of an item $A=v$ to $H$ is to see how much the difference is lifted.

Definition $3(\operatorname{DiffLift}(A=v \mid H))$ : Let $H=\left\langle P, A_{\text {diff }}=\right.$ $v_{1}\left|v_{2}, A_{\text {target }}, v_{\text {target }}\right\rangle$ be a hypothesis, $A_{\text {target }}$ be categorical, $P_{1}=P \cup\left\{A_{\text {diff }}=v_{1}\right\}$ and $P_{2}=P \cup\left\{A_{\text {diff }}=v_{2}\right\}$ be the two sub-populations of $H, A=v$ be an item not in $H$, that is, $A \neq A_{\text {diff }}, A \neq A_{\text {target }}$ and $A=v \notin P$. After adding item $A=v$ to $P$, we get two new sub-populations: $P_{1}^{\prime}=P_{1} \cup\{A=v\}$ and $P_{2}^{\prime}=P_{2} \cup\{A=v\}$. The lift of difference after adding $A=v$ to $H$ is defined as $\operatorname{DiffLift}(A=v \mid H)=\frac{p_{1}^{\prime}-p_{2}^{\prime}}{p_{1}-p_{2}}$, where $p_{i}$ is the proportion of $v_{\text {target }}$ in sub-population $P_{i}$, and $p_{i}^{\prime}$ is the proportion of $v_{\text {target }}$ in sub-population $P_{i}^{\prime}, i=1,2$.

We can divide the values of $\operatorname{DiffLift}(A=v \mid H)$ into three ranges, and each range represents a different situation.

Situation 1: $\operatorname{DiffLift}(\boldsymbol{A}=\boldsymbol{v} \mid \boldsymbol{H})>1$. The new difference is wider than the old difference and it is also of the same direction as the old difference. The larger the DiffLift, the more interesting the item. For example, in Table III, the DiffLifts of "time-of-failure=loading", "time-of-failure=in-operation", "time-of-call=outputting" are 2.05, 0, 0.05 respectively. Hence "time-of-failure=loading" is a very interesting item.

Situation 2: $0 \leq \operatorname{DiffLift}(\boldsymbol{A}=\boldsymbol{v} \mid \boldsymbol{H}) \leq 1$. The new difference is of the same direction as the old difference, but it is narrower than the old difference. The items satisfying this condition usually are not very interesting, e.g., "time-of-failure=inoperation" and "time-of-failure=outputting" in Table III.

Situation 3: $\operatorname{DiffLift}(\boldsymbol{A}=\boldsymbol{v} \mid \boldsymbol{H})<0$. The new difference is of the opposite direction of the old one. If the values of an attribute all satisfy this condition, then we have a Simpson's Paradox as in Table IV where DiffLift(stage $\left.=1 \mid H_{1}\right)=-0.683$ and DiffLift(stage $\left.=2 \mid H_{1}\right)=-0.833$.

Definition 4 (Simpson's Paradox): Given a hypothesis $H$ and an attribute $A$ not in $H$, if for every value $v$ of $A$, we have $\operatorname{DiffLift}(A=v \mid H)<0$, then we say $H$ and $A$ form a Simpson's Paradox.

We define the DiffLift of an attribute $A$ as the average of the absolute DiffLift of its attribute values.

Definition $5(\operatorname{DiffLift}(A \mid H))$ : Let $v_{1}, v_{2}, \cdots, v_{k}$ be the set of values taken by attribute $A$. Then the difference lift of $A$ with respect to $H$ is defined as $\operatorname{DiffLift}(A \mid H)=$ $\frac{\sum_{i=1}^{k}\left|\operatorname{DiffLift}\left(A=v_{i} \mid H\right)\right|}{k}$.

\section{Contribution of items and attributes}


In some cases, $\operatorname{DiffLift}(A=v \mid H)$ is not sufficient to capture all the information. Let $n_{i}$ be the size of sub-population $P_{i}$, $n_{i}^{\prime}$ be the size of sub-population $P_{i}^{\prime}, i=1,2$. If $n_{i}^{\prime}$ is extremely small compared with $n_{i}, i=1,2$, then even if $\operatorname{DiffLift}(A=v \mid H)$ is positive and very large, item $A=v$ can hardly have any material impact on $H$. We are more interested in finding those attribute values that have a big positive $\operatorname{DiffLift}(A=v \mid H)$, and are also associated with a large number of records, so that acting upon such attribute values, we are able to make much bigger impact than acting upon attribute values that are associated with few records.

If we divide sub-population $P_{1}$ into several disjoint subsets $P_{11}, P_{12}, \cdots, P_{1 k}$, then the proportion of $v_{\text {target }}$ in $P_{1}$ can be expressed as $p_{1}=\sum_{i=1}^{k} \frac{n_{1 i}}{n_{1}} p_{1 i}$, where $n_{1}$ is the size of sub-population $P_{1}, n_{1 i}$ is the size of subset $P_{1 i}$ and $p_{1 i}$ is the proportion of $v_{\text {target }}$ in subset $P_{1 i}$. Hence $\frac{n_{1 i}}{n_{1}}\left(p_{1 i}-p_{1}\right)$ can be regarded as the overall contribution of $P_{1 i}$ to $P_{1}$, denoted as Contribution $\left(P_{1 i} \mid P_{1}\right)$. We have $\sum_{i=1}^{k} \frac{n_{1 i}}{n_{1}}\left(p_{1 i}-p_{1}\right)=$ $\sum_{i=1}^{k} \frac{n_{1 i}}{n_{1}} p_{1 i}-p_{1} \frac{\sum_{i=1}^{k} n_{1 i}}{n_{1}}=0$. The contribution of $A=v$ to $H$ is determined by which one is bigger, the contribution of $P_{1}^{\prime}$ to $P_{1}$, or the contribution of $P_{2}^{\prime}$ to $P_{2}$.

Definition 6 (Contribution $(A=v \mid H)$ ): Let $H, A=v$, $P_{i}, p_{i}, P_{i}^{\prime}$ and $p_{i}^{\prime}, i=1,2$, be defined as in Definition 3. The contribution of $A=v$ to $H$ is defined as Contribution ( $A$ $=v \mid H)=\left(\frac{n_{1}^{\prime}}{n_{1}}\left(p_{1}^{\prime}-p_{1}\right)-\frac{n_{2}^{\prime}}{n_{2}}\left(p_{2}^{\prime}-p_{2}\right)\right) /\left(p_{1}-p_{2}\right)$.

If Contribution $(A=v \mid H)>0$, we say $A=v$ contributes positively to $H$; if Contribution $(A=v \mid H)<0$, we say $A=v$ contributes negatively to $H$; otherwise, we say $A=v$ makes no contributes to $H$. We define the Contribution of an attribute $A$ as the average of the absolute Contribution of its attribute values.

Definition 7 (Contribution $(A \mid H)$ ): Let $v_{1}, v_{2}, \cdots, v_{k}$ be the set of values taken by attribute $A$. Then the contribution of $A$ to $H$ is defined as Contribution $(A \mid H)=$ $\sum_{i=1}^{k}\left|\operatorname{Contribution}\left(A=v_{i} \mid H\right)\right|$.

To help users have a better understanding of the discovered hypotheses, for each significant hypothesis $H$, we identify those attributes and items that have either high DiffLift or high Contribution, and generate a ranked list of these attributes and items. We also identify the attributes that can form Simpson's Paradoxes with $H$. Users can then browse the generated information to find things that are interesting to them.

\section{E. Generalizations}

Generalization to continuous target attribute. When $A_{\text {target }}$ is continuous, we can simply use $m_{i}$ to replace $p_{i}$, and $m_{i}^{\prime}$ to replace $p_{i}^{\prime}$ in Definitions 2, 3 and 6, where $m_{i}$ and $m_{i}^{\prime}$ is the mean of $A_{\text {target }}$ in sub-population $P_{i}$ and $P_{i}^{\prime}$ respectively, $i=1,2$. If $A_{\text {target }}$ is normally distributed, we can use t-test [9] to calculate p-values; otherwise, we can use Mann-Whitney test [16].

Generalization to hypotheses involving only one sample or more than two samples. We can simply represent a hypothesis involving one sample as $H=\left\langle P, A_{\text {target }}, v_{\text {target }}\right\rangle$.
In this case, the statistics of sub-population $P$ is compared with the whole population. DiffLift and Contribution can be defined accordingly. It is also straightforward to formulate and test hypotheses involving more than two samples. A hypothesis involving $k(k>2)$ samples can be represented as $H=\left\langle P, A_{\text {diff }}=v_{1}\left|v_{2}\right| \cdots \mid v_{k}, A_{\text {target }}, v_{\text {target }}\right\rangle$. Statistical tests for more than two samples are also available. However, the analysis of hypothesis involving more than two samples is much more complicated. We leave it to our future work.

\section{F. Problem statement}

If the size of the two sub-populations under comparison is too small, statistical tests usually do not have enough power to detect the difference even if the difference is real. Hence testing hypotheses involving very small sub-populations is often futile. To save computation cost, we put a minimum support constraint min_sup on the size of the two subpopulations.

Our system requires users to supply the following parameters: (1) a minimum support threshold min_sup; (2) a maximum p-value threshold max_pvalue which indicates the level of statistical significance if one single hypothesis is tested; (3) a minimum difference threshold min_diff $f$ which reflects the level of domain significance; (4) a target attribute $A_{\text {target }}$ and a target attribute value $v_{\text {target }}$ if $A_{\text {target }}$ is categorical; (5) a set of grouping attributes $\mathcal{A}_{\text {grouping }}$, and the grouping attributes must be categorical. Users can set the parameters based on their requirements and domain knowledge. The last parameter is optional. If users do not specify the grouping attributes, then we use all the categorical attributes in the given dataset as grouping attributes.

Exploratory hypothesis testing. Given a dataset $D$ and the above parameters, the hypothesis testing task aims to find all the hypotheses $H=\left\langle P, A_{\text {diff }}=v_{1} \mid v_{2}, A_{\text {target }}, v_{\text {target }}\right\rangle$ satisfying the following conditions:

1) $A_{\text {diff }} \in \mathcal{A}_{\text {grouping }}$, and $\forall$ item $A=v$ in $P, A \in$ $\mathcal{A}_{\text {grouping. }}$.

2) $\sup \left(P_{1}\right) \geq$ min_sup, sup $\left(P_{2}\right) \geq$ min_sup, where $P_{1}=P \cup\left\{A_{\text {diff }}=v_{1}\right\}, P_{2}=P \cup\left\{A_{\text {diff }}=v_{2}\right\}$.

3) p-value $(H) \leq$ max _pvalue.

4) If $A_{\text {target }}$ is categorical, then $\left|p_{1}-p_{2}\right| \geq$ min_diff, where $p_{i}$ is the proportion of $v_{\text {target }}$ in sub-population $P_{i}, i=1,2$.

If $A_{\text {target }}$ is continuous, then $\left|m_{1}-m_{2}\right| \geq m_{i n}$ diff , where $m_{i}$ is the mean of $A_{\text {target }}$ in sub-population $P_{i}$, $i=1,2$.

Hypothesis analysis. For each significant hypothesis $H$, the hypothesis analysis task generates the following information for further analysis: 1) The set of Simpson's Paradoxes formed by $H$ with attributes not in $H$. 2) The list of items not in $H$ ranked in descending order of $\operatorname{DiffLift}(A=v \mid H)$ and Contribution $(A=v \mid H)$ respectively. 3) The list of attributes not in $H$ ranked in descending order of $\operatorname{DiffLift}(A \mid H)$ and Contribution $(A \mid H)$ respectively. 


\section{Automatic HYPOTHESIS TESTING AND ANALYSIS}

We generate hypotheses in two steps. First, we generate large sub-populations using existing frequent pattern mining techniques. Generating large sub-populations containing no less than min_sup records is equivalent to mining frequent patterns with support no less than min_sup. Many efficient algorithms have been developed for mining frequent patterns [8]. They can be used to generate large sub-populations. In the second step, we pair the large sub-populations up to form tentative hypotheses. The number of large sub-populations generated may be very large, so we need to store the patterns in a structure that supports efficient pattern retrieval. A suitable candidate for this task is the CFP-tree structure [15].

In the rest of this section, we first present the hypotheses generation and analysis algorithms without restricting ourselves to any frequent pattern mining algorithm or any structure for storing patterns, and then describe how the CFPtree structure can be utilized to improve the efficiency of the algorithms.

\section{A. Finding large sub-populations}

Existing frequent pattern mining algorithms generate only frequent patterns and their supports. Additional information is needed for calculating $\mathrm{p}$-values and for analyzing significant hypotheses. We modified the frequent pattern mining algorithm used in our system as follows.

We collect more statistics of the large sub-populations besides the support of the patterns defining them for the calculation of p-values. We take the two commonly used tests, $\chi^{2}$-test and t-test, as examples. Let $P$ be a frequent pattern. When $\chi^{2}$-test is used, we need to collect $\sup (P)$ and $\sup \left(P \cup\left\{A_{\text {target }}=v_{\text {target }}\right\}\right)$ to get the proportion of $A_{\text {target }}=v_{\text {target }}$ in sub-population $P$. When t-test is used, we need to get the mean $m_{P}$ and the standard deviation $s_{P}$ of $A_{\text {target }}$ in sub-population $P$. These two statistics are defined as follows: $m_{P}=\frac{\sum_{i=1}^{s u p(P)} v_{i}}{\sup (P)}$ and $s_{P}=\sqrt{\frac{\sum_{i=1}^{\sup (P)}\left(v_{i}-m_{P}\right)^{2}}{\sup (P)-1}}$ $=\sqrt{\frac{\sum_{i=1}^{s u p(P)} v_{i}^{2}-\sup (P) \cdot m_{P}^{2}}{\sup (P)-1}}$, where $v_{i}$ is the $A_{\text {target }}$ value of the $i$-th record in sub-population $P$. Hence we need to collect $\sup (P), \sum_{i=1}^{\sup (P)} v_{i}$ and $\sum_{i=1}^{\sup (P)} v_{i}^{2}$ to calculate $m_{P}$ and $s_{P}$. All these information can be collected as we count the support of pattern $P$. No additional scan of data is needed.

When analyzing a significant hypothesis $H$, we divide the two sub-populations of $H$ into finer sub-populations by adding an item to the defining patterns of the two sub-populations. The resultant patterns may not be frequent. Hence we need to generate not only frequent patterns, but also some infrequent patterns to make sure that all the information needed for analyzing a hypothesis is available. The generated infrequent patterns are the immediate super-patterns of some frequent patterns. In the original algorithm, a pattern is not extended if it is not frequent. In the modified version, an infrequent pattern is still extended if it has at least one frequent immediate subpattern. That is, a pattern is not extended only if all of its immediate sub-patterns are infrequent. We explore the search space in a way such that the sub-patterns of a pattern are generated before the pattern itself. This exploration strategy has been used in mining frequent generators [14].

\section{B. Generating tentative hypotheses}

Given a hypothesis $H=\left\langle P, A_{\text {diff }}=v_{1}\right| v_{2}, A_{\text {target }}$, $\left.v_{\text {target }}\right\rangle$, the defining patterns of the two sub-populations of $H$ contain one more item than the context pattern $P$, so they are immediate super-patterns of $P$. We generate tentative hypotheses as follows. For each frequent pattern $P$, we use it as a context and retrieve all of its frequent immediate superpatterns. We then group these super-patterns of $P$ based on the attributes not in $P$. Patterns that have the same attribute not in $P$ are placed in the same group. Patterns in the same group are then paired up to form hypotheses. The pseudo-codes for generating hypotheses are shown in Algorithm 1.

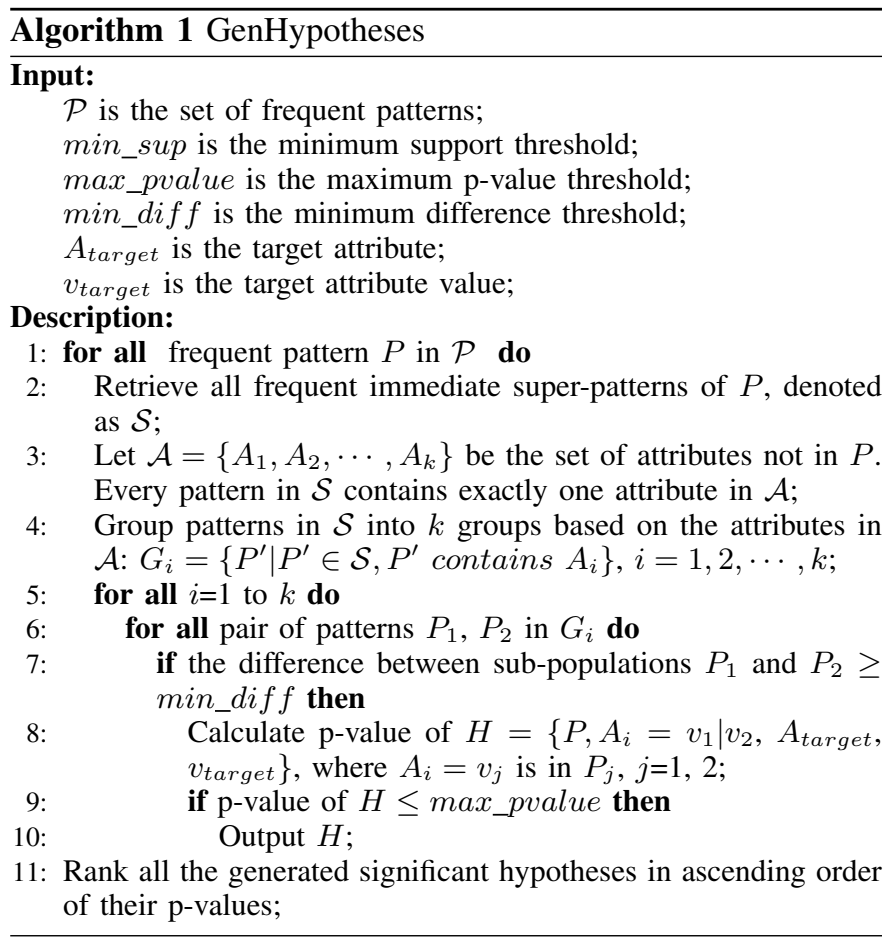

\section{Generating information for hypothesis analysis}

If a hypothesis is statistically significant, we generate information for its further analysis. The pseudo-codes are shown in Algorithm 2. Given a significant hypothesis $H$ with two sub-populations $P_{1}$ and $P_{2}$, we retrieve the immediate superpatterns of $P_{1}$ and $P_{2}$ (line 1-2). Let $P_{i}^{\prime}$ be an immediate superpattern of $P_{i}, i=1,2$. If $P_{1}^{\prime}$ and $P_{2}^{\prime}$ contain the same item that appears in neither $P_{1}$ nor $P_{2}, P_{1}^{\prime}$ and $P_{2}^{\prime}$ form a pair $\left(P_{1}^{\prime}, P_{2}^{\prime}\right)$. We group these pairs based on the attributes not in $H$ (line 3 ). For each item $A=v$ that is not in $H, \operatorname{DiffLift}(A=v \mid H)$ and Contribution $(A=v \mid H)$ are calculated using the statistics of $P_{1}, P_{2}, P_{1} \cup\{A=v\}$ and $P_{2} \cup\{A=v\}$ (line 4-7). The DiffLift and Contribution of each attribute are calculated using that of its items (line 8-9). Simpson's Paradoxes are generated based on DiffLift (line 10-11). After all the information for analysis are generated, we then output lists of items and attributes 


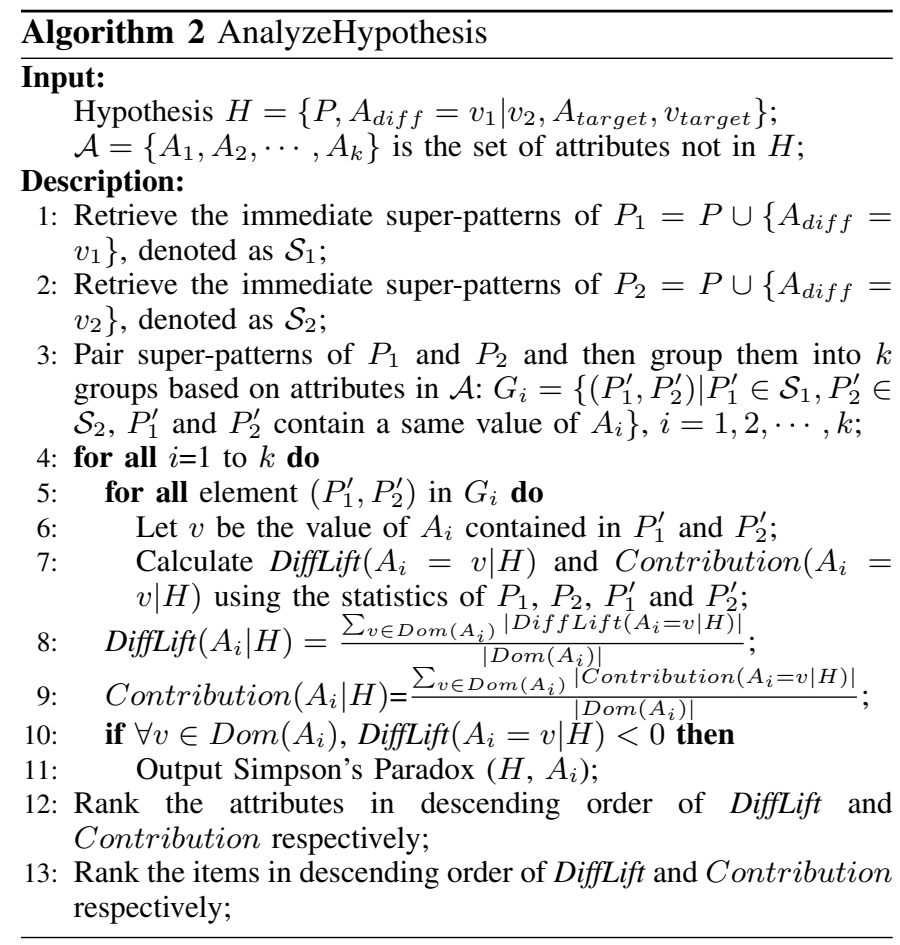

ranked in descending order of DiffLift or Contribution (line 12-13). At line 8-10, $\operatorname{Dom}\left(A_{i}\right)$ is the set of values taken by attribute $A_{i}$.

\section{Implementation}

The implementation of the operations in Algorithm 1 and 2 is straightforward except that of retrieving immediate superpatterns. When the number of patterns generated is very large, the cost for retrieving immediate super-patterns can be very high. We use the CFP-tree structure [15] to speed-up this operation. Here we introduce the basic features of the CFPtree structure that are relevant to this work. For more details on the CFP-tree structure, please refer to the original paper.

CFP-tree is a very compact structure for storing patterns. It allows different patterns to share the storage of their prefixes as well as suffixes. It has been shown that a CFP-tree storing all frequent patterns can be orders of magnitude smaller than its corresponding flat representation [15]. CFP-tree supports efficient exact match and subset/superset search, which are repeatedly used in our algorithms.

Given a database $D$, the set of possible patterns in $D$ can be represented as a set-enumeration tree. Figure 1 shows an example set-enumeration tree on a dataset with three attributes $a, b$ and $c$. The three attributes are sorted lexicographically. Each attribute $x$ has two values: $x_{1}$ and $x_{2}, x=a, b, c$. For brevity, we use $x_{i}$ to represent item $x=x_{i}$, where $x=a, b$, $c$ and $i=1,2$. A node in the set-numeration tree represents a pattern. Each pattern can be extended only by the items belonging to the attributes before it. For example, items $b_{1}$ and $b_{2}$ can be used to extend pattern $\left\{c_{1}\right\}$, but they cannot be used to extend pattern $\left\{a_{1}\right\}$. The set of items that can be used to extend an pattern $P$ are called candidate extensions of $P$, denoted as cand_exts $(P)$.

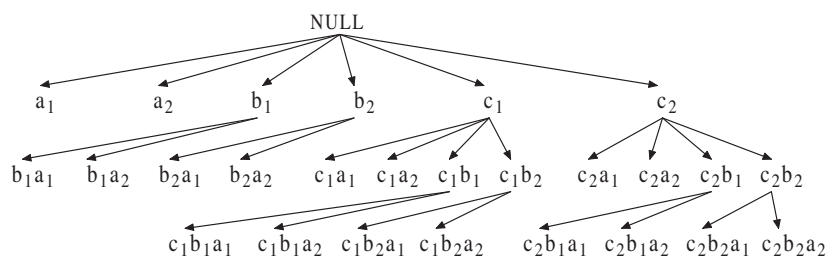

Fig. 1. An example search space tree with 3 attributes $a, b$, and $c$. Each attribute $x$ has two values: $x_{1}$ and $x_{2}, x=a, b, c$

The CFP-tree structure [15] is a prefix-trie structure. It is similar to the set-enumeration tree but it allows suffix sharing. Figure 2(c) shows an example CFP-tree, and it stores the set of frequent patterns in the dataset shown in Figure 2(a). The dataset has four attributes: $a, b, c$ and $d$. The minimum support threshold is set to 3 . The set of frequent patterns are shown in Figure 2(b). For brevity, a frequent pattern $\left\{i_{1}, i_{2}, \cdots, i_{m}\right\}$ with support $n$ is represented as $i_{1} i_{2} \cdots i_{m}: n$.

Each node in a CFP-tree consists of a number of entries. Each entry contains exactly one item if the node has multiple entries. An entry can contain multiple items if it is the only entry of its parent. For example, node 7 in Figure 2(c) has only one entry and this entry contains two items $c_{2}$ and $a_{1}$. A path starting from an entry in the root node represents one or more frequent patterns and these patterns have the same support. Let $E$ be an entry and $P$ be a pattern represented by the path from root to $E$. Entry $E$ stores several pieces of information: (1) $n$ items ( $n \geq 1)$, (2) the support of $P$ and other information for calculating p-values, and (3) a pointer pointing to the child node of $E$.

|
\begin{tabular}{|l|l|l|l|}
\hline $\mathbf{1}$ & $a_{1}, b_{1}, c_{2}, d_{1}$ & 6 & $a_{1}, b_{2}, c_{2}, d_{2}$ \\
\hline $\mathbf{2}$ & $a_{2}, b_{1}, c_{1}, d_{1}$ & 7 & $a_{1}, b_{2}, c_{1}, d_{2}$ \\
\hline $\mathbf{3}$ & $a_{1}, b_{2}, c_{1}, d_{2}$ & 8 & $a_{1}, b_{1}, c_{2}, d_{1}$ \\
\hline $\mathbf{4}$ & $a_{1}, b_{1}, c_{2}, d_{1}$ & 9 & $a_{2}, b_{1}, c_{2}, d_{2}$ \\
\hline $\mathbf{5}$ & $a_{2}, b_{2}, c_{2}, d_{2}$ & & \\
\hline$a_{1}: 6, a_{2}: 3, b_{1}: 5, b_{2}: 4, c_{1}: 3, c_{2}: 6, d_{1}: 4, d_{2}: 5$ \\
\hline$b_{1} a_{1}: 3, b_{2} a_{1}: 3, c_{2} a_{1}: 3, c_{2} b_{1}: 4, d_{1} b_{1}: 4$, \\
$d_{1} a_{1}: 3, d_{1} c_{2}: 3, d_{2} a_{1}: 3, d_{2} b_{2}: 4, d_{2} c_{2}: 3$, \\
\hline$c_{2} b_{1} a_{1}: 3, d_{1} b_{1} a_{1}: 3, d_{1} b_{1} c_{2}: 3, d_{1} c_{2} a_{1}: 3$, \\
$d_{2} b_{2} a_{1}: 3$ \\
\hline$d_{1} b_{1} c_{2} a_{1}: 3$ \\
\hline
\end{tabular}

(a) Dataset

(b) Frequent patterns

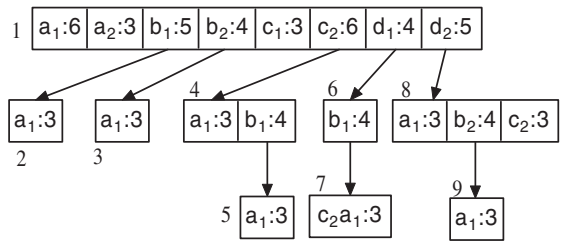

(c) A CFP-tree

Fig. 2. An example CFP-tree

The CFP-tree structure allows different patterns to share the storage of their prefixes as well as suffixes, which makes it a very compact structure for storing frequent patterns. Prefix sharing is easy to understand. For example, patterns $\left\{c_{2}, a_{1}\right\}$ 
and $\left\{c_{2}, b_{1}\right\}$ share the same prefix $c_{2}$ in Figure 2(c). Suffix sharing is not as obvious as prefix sharing. Suffix sharing occurs when a pattern has the same support as its prefix. Let $P$ be a pattern. If there exists a candidate extension $i$ of $P$ such that $i$ occurs in every record containing $P$, that is, $\sup (P \cup\{i\})=\sup (P)$, then for every other candidate extension $i^{\prime}$ of $P$, we have $\sup \left(P \cup\{i\} \cup\left\{i^{\prime}\right\}\right)=\sup \left(P \cup\left\{i^{\prime}\right\}\right)$. In this case, pattern $P \cup\{i\}$ and $P$ share suffixes. In Figure 2(a), item $b_{1}$ appears in every record containing pattern $\left\{d_{1}\right\}$. Therefore, patterns $\left\{d_{1}\right\}$ and $\left\{d_{1}, b_{1}\right\}$ share suffixes, which is node 7. Node 7 represents 6 patterns: $\left\{d_{1}, c_{2}\right\},\left\{d_{1}, a_{1}\right\}$, $\left\{d_{1}, c_{2}, a_{1}\right\},\left\{d_{1}, b_{1}, c_{2}\right\},\left\{d_{1}, b_{1}, a_{1}\right\},\left\{d_{1}, b_{1}, c_{2}, a_{1}\right\}$.

Suffix sharing is implemented by singleton nodes in a CFP-tree. More specifically, if patterns $P$ and $P \bigcup X$, where $X \subseteq$ cand_exts $(P)$, satisfy $\sup (P \cup X)=\sup (P)$, then the child node of $P$ contains only one entry and the set of items of the entry is $X$. For example, the child node of $\left\{d_{1}\right\}$ is a singleton node containing item $b_{1}$. If there are more than one singleton node on a path, then the space saved by suffix sharing multiplies. The space saved by suffix sharing is dramatic. A CFP-tree storing a complete set of frequent patterns can be orders of magnitude smaller than the corresponding flat representation [15].

\section{Constructing a CFP-tree}

Constructing a CFP-tree from a dataset is almost the same as mining frequent patterns from the dataset. The main difference is the output format: a CFP-tree or a flat file. Since we store patterns in a CFP-tree, we use the CFP-tree construction algorithm described in [15] to find large sub-populations.

Besides the two modifications described in Section III-A, we made another modification to the original CFP-tree construction algorithm. In the original algorithm, items are sorted in descending order of their support. In the modified version, items that are of the same attribute are sorted to be adjacent to each other, and their attributes are sorted in ascending order of the number of distinct values. The benefit of this sorting strategy is that in Algorithm 1 and 2, the retrieved immediate super-patterns of a pattern are already grouped based on the attributes not in the pattern when they are retrieved. The cost for grouping them is thus saved.

\section{Query processing on the CFP-tree structure}

The CFP-tree structure has an important property that makes it very suitable for exact match and subset/superset search.

Property 1: Let cnode be a non-singleton CFP-tree node and $E$ be an entry of cnode. The item of $E$ can appear only in the subtrees pointed by entries after $E$ in cnode.

For example, in node 1 of Figure 2(c), item $c_{2}$ can appear in the subtrees pointed by $d_{1}$ and $d_{2}$, but it cannot appear in the subtrees pointed by $a_{1}, a_{2}, b_{1}, b_{2}$ and $c_{1}$. Based on this property, a sub-pattern of a pattern is either a prefix of the pattern or appears on the left of the pattern; a super-pattern of a pattern appears either in the subtree pointed by the pattern or on the right of the pattern.

We need to perform two types of queries on a CFP-tree: searching for the immediate sub-patterns of a pattern and searching for the immediate super-patterns of a pattern. The former is used during the generation of large sub-populations for checking whether an infrequent pattern has a frequent immediate sub-pattern. The latter is used for hypothesis generation and analysis. We convert both queries to exact match. A pattern $P$ has $|P|$ immediate subsets, so searching for the immediate sub-patterns of a pattern $P$ can be converted to $|P|$ exact matches. For immediate super-pattern queries, only one additional item is allowed. Hence once there is a mismatch at an entry, i.e., the item in the entry is the additional item, then the subsequent searching in the subtree rooted at that entry must be exact match.

Algorithm 3 shows the pseudo-codes for exact match. When it is first called, $P$ is the pattern to be searched, and cnode is the root node of the CFP-tree. At each node being visited, we need to search further in at most one subtree of the node, and this subtree is pointed by the rightmost entry whose item is contained in the pattern to be searched. For example, to search pattern $P=\left\{c_{2}, a_{1}\right\}$ in the CFP-tree in Figure 2(c), we only need to visit the subtree pointed by item $c_{2}$ in node 1 because the subtrees pointed by entries before $c_{2}$ do not contain $c_{2}$, and the entries after $c_{2}$ contain items that are not in $P$.

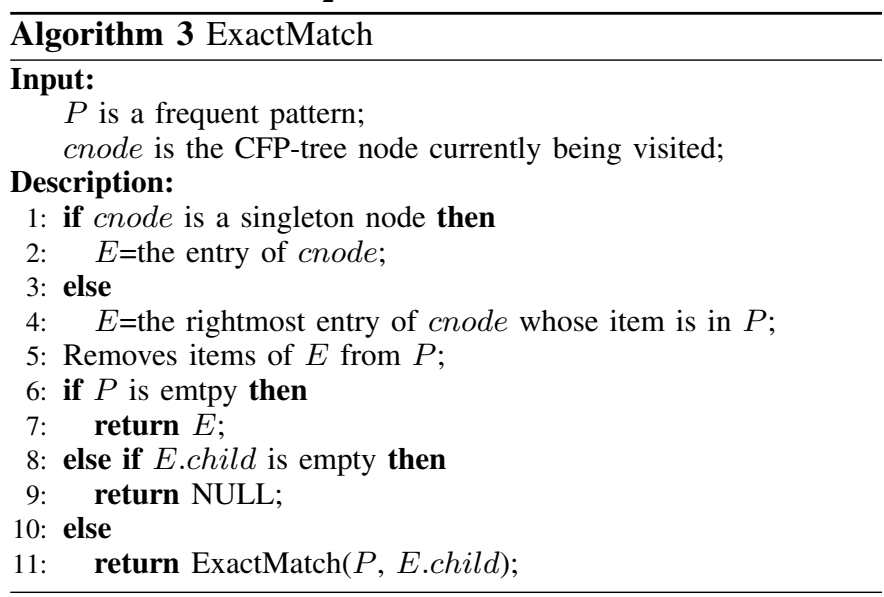

\section{A Performance Study}

In this section, we study the efficiency of the proposed algorithms, and demonstrate the usefulness of the generated results via two case studies. We conducted the experiments on a PC with 2.33Ghz Intel(R) Core(TM) Duo CPU and 3.25GB memory. The operating system is Windows XP professional. Our algorithms were implemented in $\mathrm{C}++$ and complied using Visual Studio 2005.

\section{TABLE V}

DATASETS

\begin{tabular}{|c|c|c|c|c|}
\hline Datasets & \#instances & $\begin{array}{c}\text { \#continuous } \\
\text { attributes }\end{array}$ & $\begin{array}{c}\text { \#categorical } \\
\text { attributes }\end{array}$ & $\begin{array}{c}A_{\text {target }} / \\
v_{\text {target }}\end{array}$ \\
\hline adult & 48842 & 6 & 9 & class=>50K \\
mushroom & 8124 & 0 & 23 & class=poisonous \\
DrugTestI & 141 & 13 & 74 & $\operatorname{logAUCT}$ \\
DrugTestII & 138 & 13 & 74 & $\operatorname{logAUCT}$ \\
\hline
\end{tabular}

We used four datasets in our experiments: adult, mushroom, DrugTestI and DrugTestII. Table V shows some statistics of these four datasets. Datasets adult, and mushroom are obtained from UCI machine learning repository(http://archive. ics.uci.edu/ml/). Datasets DrugTest I and DrugTest II are 
two pharmacogenetics datasets that study the associations between mutations in several genes and drug responses. Single Nucleotide Polymorphisms (SNPs)—single DNA sequence mutations - in the target genes were genotyped in volunteers from several populations.

\section{A. Mining efficiency}

The performance of the CFP-tree construction algorithm, denoted as CFP-growth, is comparable to the state-of-the-art frequent pattern mining algorithms [15]. The first experiment studies the impact of generating infrequent patterns on the efficiency of CFP-growth and the size of the generated CFP-tree. Figure 3 shows the results on datasets adult and mushroom. The target attributes are shown in the last column of Table V. All categorical attributes except $A_{\text {target }}$ are used as grouping attributes.

On adult, the running time is almost the same for both cases, while the CFP-tree size doubles when infrequent patterns are included. On mushroom, the running time increases greatly when infrequent patterns are included, but the size of the CFPtree increases just slightly. The reason being that mushroom is much denser than adult, and the average pattern length in mushroom is much longer than that in adult. There is lots of sharing among patterns in mushroom, hence the increase in tree size is not obvious when infrequent patterns are included. However, the CFP-tree constructed on mushroom is much larger than that on adult, so the cost for subset checking is much more expensive, which increases the running time significantly. We have also studied the size of the conditional databases generated during the mining process, and we found that the size is almost the same in the two cases on both datasets.

In practice, we do not expect users generate very long patterns as the contexts of hypotheses because the contexts would be too specific. If we set a length limit to the patterns, the increase in running time and tree size should not be dramatic when infrequent patterns are included.

We also compared the running time of CFP-growth with FP-growth [10]. On adult, FP-growth is faster than CFPgrowth. However, most of the time of CFP-growth is spent on data preprocessing such as mapping attribute values to items (integers) and converting the original dataset to a transaction dataset. On mushroom, CFP-growth is faster than FP-growth when min_sup is low even when CFP-growth generates infrequent patterns. This is because mushroom is very dense and many patterns are generated. FP-growth has a much longer output time than CFP-growth since it stores patterns in a flat format.

The second experiment studies the efficiency of the algorithms for hypotheses generation and analysis. The target attribute on each dataset is shown in the last column of Table V. Table VI shows that in most cases, identifying significant hypotheses took less than 1 second, and the average time for generating the information for analyzing one single hypothesis is below 0.01 second. The above results suggest that it is possible to identify significant hypotheses and analyze them

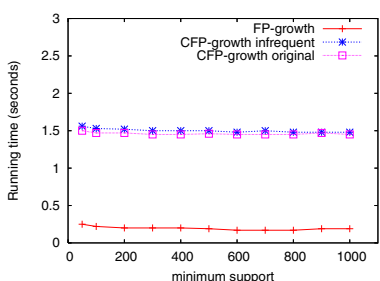

(a) adult: runtime

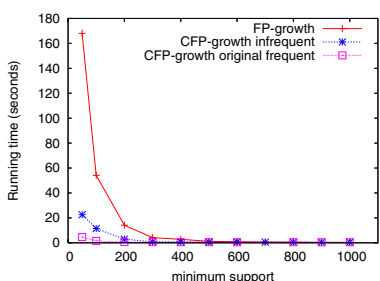

(c) mushroom: runtime
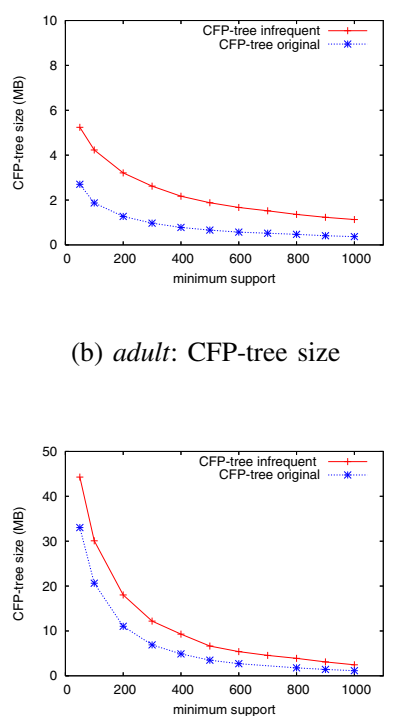

(d) mushroom:CFP-tree size (b) adult: CFP-tree size

Fig. 3. Running time of the CFP-tree construction algorithm and the size of CFP-tree with and without generating infrequent patterns that have at least one frequent immediate sub-pattern. "CFP-growth original" represents the original CFP-tree construction algorithm. "CFP-growth infrequent" refers to the modified algorithm. "CFP-tree original" refers to the CFP-tree storing only frequent patterns. "CFP-tree infrequent" refers to the CFP-tree storing both frequent and infrequent patterns.

on demand given that frequent patterns and their immediate infrequent super-patterns have been materialized. The high efficiency of the algorithms for hypotheses generation and analysis is attributed to the high efficiency of the CFP-tree structure in supporting superset queries.

The last four columns of Table VI show the number of tests performed and the number of significant hypotheses generated. On the two large datasets mushroom and adult, most of the significant hypotheses are still statistically significant after correction. On the two DrugTest datasets, many hypotheses become insignificant after correction, especially when Bonferroni correction is used. This may be due to two reasons: one is that the number of records in the two DrugTest datasets is too small, and the other reason is that Bonferroni correction is too restrictive.

Our algorithms need to access the original datasets only in the large sub-population generation phase using frequent pattern mining techniques, so our algorithms can ran on large datasets that the state-of-the-art frequent pattern mining algorithms can handle. The CFP-tree storing the sub-populations may be large, but the cost for retrieving patterns from a CFPtree is not sensitive to the size of the CFP-tree. Hence the running time of Algorithm 1 is not quadratic to the number of frequent patterns even though it needs to pair frequent patterns up for comparison. We observed that the running time of Algorithm 1 is nearly linear to the number of frequent patterns. The running time of Algorithm 2 is also scalable to the size of the CFP-tree. 
TABLE VI

RUNNING TIME (MEASURED IN SECONDS) AND NUMBER OF SIGNIFICANT HYPOTHESES GENERATED. max_pvalue is set to 0.05 on all datasets. "GenH": time for testing all hypotheses; “AnalyzeH”: time for analysis of all significant hypotheses; "AvgAnalyzeT”: average time for analyzing a single

hypothesis; "\#test": total number of tests performed; “\#SignH”: \#significant hypotheses with p-value $\leq$ max_pvalue; "\#SignH_BC": \#significant hypotheses with p-value $\leq$ max_pvalue/\#test (Bonferroni correction); “\#SignH_FDR0.05”: \#significant hypotheses when FDR is set at 0.05 (Benjamini and Hochberg's method).

\begin{tabular}{|c|c|c|c|c|c|c|c|c|c|}
\hline Datasets & min_sup & min_diff & GenH & AnalyzeH & AvgAnalyzeT & \#tests & \#SignH & \#SignH_BC & \#SignH_FDR0.05 \\
\hline adult & 500 & 0.05 & $0.42 \mathrm{sec}$ & $6.30 \mathrm{sec}$ & $0.0015 \mathrm{sec}$ & 5593 & 4258 & 3929 & 4257 \\
adult & 100 & 0.05 & $2.69 \mathrm{sec}$ & $37.39 \mathrm{sec}$ & $0.0014 \mathrm{sec}$ & 41738 & 26095 & 16345 \\
mushroom & 500 & 0.1 & $0.67 \mathrm{sec}$ & $19.00 \mathrm{sec}$ & $0.0020 \mathrm{sec}$ & 16400 & 9323 & 9244 \\
mushroom & 200 & 0.1 & $5.45 \mathrm{sec}$ & $123.47 \mathrm{sec}$ & $0.0020 \mathrm{sec}$ & 103025 & 61429 & 57798 \\
DrugTestI & 20 & 0.5 & $0.06 \mathrm{sec}$ & $0.06 \mathrm{sec}$ & $0.0031 \mathrm{sec}$ & 3627 & 20 & 1 \\
DrugTestII & 20 & 0.5 & $0.08 \mathrm{sec}$ & $0.30 \mathrm{sec}$ & $0.0031 \mathrm{sec}$ & 4441 & 97 & 61429 \\
\end{tabular}

TABLE VII

AN EXAMPLE OF A SIGNIFICANT HYPOTHESIS IDENTIFIED FROM DATASET DrugTestII AND POSSIBLE REASONS BEHIND IT. SNP_OATPB_14 is $a$ SNP at locus 14 of gene OATPB.

\begin{tabular}{|c|c|c|c|c|c|}
\hline ID & Context & Comparing Groups & sup & mean & p-value \\
\hline$H$ & $\int$ & Ethnic_Group=Japanese & 25 & 5.256 & $<\Omega \cap \Omega 1$ \\
\hline$\Pi_{1}$ & \{\} & Ethnic_Group=Caucasian & 22 & 4.750 & .001 \\
\hline
\end{tabular}

(a) An example of significant hypotheses in dataset DrugTest II

\begin{tabular}{|c|l|l|c|c|}
\hline Context & Extra Attribute & Comparing Groups & sup & mean \\
\hline \multirow{3}{*}{} & \multirow{2}{*}{ SNP_OATPB_14=0 } & Ethnic_Group=Japanese & 7 & $\mathbf{4 . 7 4 9}$ \\
\cline { 2 - 5 } & & Ethnic_Group=Caucasian & 21 & $\mathbf{4 . 7 8 5}$ \\
\cline { 2 - 6 } & \multirow{2}{*}{ SNP_OATPB_14=1 } & Ethnic_Group=Japanese & 17 & $\mathbf{5 . 4 8 9}$ \\
& & Ethnic_Group=Caucasian & 1 & $\mathbf{4 . 0 0 9}$ \\
\hline
\end{tabular}

(b) The attribute with the highest contribution with respect to $H_{1}$.

\section{B. Case study I: DrugTestII}

The objective of case study I is to demonstrate the ability of the proposed system in identifying significant hypotheses and the reasons behind them. This experiment is conducted on DrugTestII. We choose continuous attribute "logAUCT" as the target attribute, and it measures the responses of the individuals to the drug.

Table 6(a) shows a significant hypothesis identified from dataset DrugTestII. Hypothesis $H_{1}$ indicates that Japanese have higher $\log$ AUCT than Caucasian on average. Table 6(b) shows the SNP with the highest contribution with respect to $H_{1}$. When SNP_OATPB_14=0, the two ethnic groups have similar $\log$ AUCT values. However, when SNP_OATPB_14 $=1$, Japanese individuals have exceptionally high $\log$ AUCT, and the number of Japanese with SNP_OATPB_14=1 is quite large.

\section{Case study II: adult}

Case study II is conducted on dataset adult. It demonstrates the ability of the proposed system in finding interesting hypotheses and Simpson's Paradoxes behind them. Attribute "class" is chosen as the target attribute, and " $>50 \mathrm{~K}$ " is chosen as the target value.

Table 7(a) shows two example significant hypotheses observed in adult. Hypothesis $H_{1}$ implies that among white people, craft repairers earn more than administration clerks. However, if we take a further look by dividing the records using the "Sex" attribute, we observe the opposite phenomenon in both "male" white people and "female" white people. That is, as shown in Table 7(b), for both sexes, craft repairers actually earn less than administration clerks. This Simpson's Paradox is caused by the fact that white men earn much more than white women on average, and many more white men work as craft repairers than white women. Therefore, the true hypothesis should be "white men earn more than white women on average", and this hypothesis is also detected by our system $\left(H_{2}\right.$ in Table 7(a)).

Simpson's Paradoxes are caused by confounding factors that have associations with both the comparing attribute and the target attribute. In Table 7 , the confounding factor is attribute "Sex". If a hypothesis $H=\left\langle P, A_{\text {diff }}=v_{1}\right| v_{2}$, $\left.A_{\text {target }}, v_{\text {target }}\right\rangle$ and an attribute $A$ form a Simpson's Paradox, it suggests two things: one is that $H$ may be wrong, and the other is that $H^{\prime}=\left\langle P, A=v_{1}|\cdots| v_{k}, A_{\text {target }}, v_{\text {target }}\right\rangle$ may be a more interesting hypothesis than $H$, where $v_{1}, \cdots, v_{k}$ are the set of values taken by attribute $A$.

\section{RELATED WORK}

Exploratory hypothesis testing needs to explore a large space of tentative hypotheses. We employ frequent pattern mining techniques for efficient exploration. Frequent pattern mining was first proposed by Agrawal et al.[2] in 1993, and it has become an important problem in the data mining area since then. Many efficient algorithms have been proposed. A frequent itemset mining implementations repository has been set up [8](http://fimi.cs.helsinki.fi/src/). Recently, Kirsch et al. [11] identify a threshold $s^{*}$ such that the set of patterns with support at least $s^{*}$ can be flagged as statistically significant with a small false discovery rate. Their work focuses on the statistical significance of the frequency of the patterns, while our work studies the statistical significance of the difference between two sub-populations.

Frequent patterns can be used to generate association rules. An association rule is of the form: $X \Rightarrow Y$, and it means that 
TABLE VIII

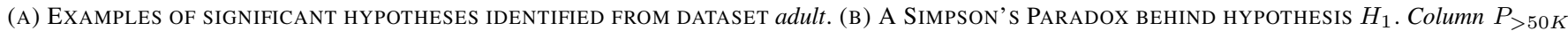
is the proportion of instances with annual income $>50 \mathrm{~K}$.

\begin{tabular}{|c|c|c|c|c|c|}
\hline ID & Context & Comparing Groups & sup & $P_{>50 K}$ & p-value \\
\hline$H_{1}$ & $\mathrm{Rac}$ & $\begin{array}{l}\text { Occupation }=\text { Craft-repair } \\
\text { Occupation }=\text { Adm-clerical }\end{array}$ & $\begin{array}{l}3694 \\
3084\end{array}$ & $\begin{array}{l}22.84 \% \\
14.23 \%\end{array}$ & \\
\hline$H_{2}$ & Race $=$ & $\begin{array}{l}\text { Sex=Male } \\
\text { Sex=Female }\end{array}$ & $\begin{array}{c}19174 \\
8642\end{array}$ & $\begin{array}{l}31.76 \% \\
11.90 \%\end{array}$ & $\leq 1.00 \mathrm{E}-08$ \\
\hline
\end{tabular}

(a) Examples of significant hypotheses in dataset adult

\begin{tabular}{|c|l|l|c|c|}
\hline Context & Extra Attribute & Comparing Groups & sup & $P_{>50 K}$ \\
\hline \multirow{3}{*}{ Race = White } & \multirow{2}{*}{ Sex = Male } & Occupation = Craft-repair & 3524 & $\mathbf{2 3 . 5 \%}$ \\
\cline { 2 - 5 } & & Occupation = Adm-clerical & 1038 & $\mathbf{2 4 . 2 \%}$ \\
\cline { 2 - 5 } & \multirow{2}{*}{ Sex = Female } & Occupation = Craft-repair & 107 & $\mathbf{8 . 8 \%}$ \\
& Occupation = Adm-clerical & 2046 & $\mathbf{9 . 2 \%}$ \\
\hline
\end{tabular}

(b) A Simpson's Paradox behind $H_{1}$.

if the left hand side $X$ occurs, then the right hand side $Y$ is also very likely to occur. An association rule $X \Rightarrow Y$ can be viewed as a special case of hypotheses, i.e., it compares the proportion of $Y$ in sub-population $X$ with that in the general population. When pattern $X$ contains multiple items, users can know the combined effect of these items, but it is hard for them to conjecture which item contributes to the difference. In exploratory hypothesis testing, we know explicitly which attribute is underlying the difference. The two approaches can be combined to have a more comprehensive view of the data.

Association rule mining algorithms often produce a large number of rules, and not all of them are interesting. Various interestingness measures have been proposed to capture the interestingness of rules. Tan et al.[20] and Geng et al.[7] surveyed various measures proposed in the literature. Many existing rule interestingness measures involve no comparison, and they look at one rule at a time. Exploratory hypothesis testing compares the difference between rules to find deviations. We also present the findings in the form of comparison (hypotheses), which allows users to look at the data from another perspective. Furthermore, we do not stop at simply comparing two rules. We also investigate the reasons behind the difference and look at issues like Simpson' Paradox.

Some interestingness measures involve comparison [18], [12], and they compare rules with existing knowledge or expectations of users. The main difficulty faced by this approach is that there is no one general method that can represent all types of knowledge, and it is often very tedious and difficult for users to specify everything they know. Contrast pattern mining also involves comparison, but it compares the statistics of a single pattern in predefined classes [3], [5]. They are more like a special type of association rules. There is no comparison between different patterns.

An interesting hypothesis consists of two rules that are significantly different. These two rules are usually ranked far apart using existing rule interestingness measures. Hence it is very tedious and time-consuming for users to manually identify interesting hypotheses from a large collection of rules, even when rules are ranked. The situation may get worse when some rules are discarded because they fail to pass the interestingness threshold. Therefore, rule ranking methods cannot be directly used for exploratory hypothesis testing.

One work that is the most related to ours is Freitas' work[6]. This work compares the class label of a rule with that of its minimum generalizations (sub-patterns), and uses the proportion of the minimum generalizations that have a different class label as a surprisingness measure. The outputs of the work are still rules. It is difficult for users to conjecture with respect to which minimum generalization, the rule is surprising. Another difference is that this work compares rules whose transaction sets have subset-superset relationships, while our work compares rules whose transaction sets have no overlap. We also provide the statistical evidence of the results, which has been largely overlooked in the past. In the same paper, Freitas also proposes an algorithm to identify Simpson's Paradox based on the change of class labels. We find Simpson's Paradox in a different situation, and we use it to identify spurious hypotheses. Freitas gave the sketches of his algorithms, but he did not discuss how to implement them efficiently. We give an efficient implementation of our algorithms. We believe that our techniques can also be applied to Freitas' work.

Recently, Liu et al.[13] developed a system called Opportunity Map, which casts rule analysis as OLAP operations and general impression mining. Users can use OLAP operations to explore rules. The same group of authors later found that although the operations on rule cubes are flexible, each operation is primitive and has to be initiated by the user. Finding a piece of actionable knowledge typically involves many operations and intense visual inspections. To solve this problem, Zhang et al.[22] proposed the idea of identifying actionable knowledge via automated comparison. In their approach, users need to manually select two attribute values for comparison, and the system then ranks all the other attributes based on their levels of interestingness. What they do is similar to the hypothesis analysis step in our approach, but the hypotheses in their system are provided by users manually instead of being generated automatically as in our approach. They do not identify Simpson's Paradoxes either.

\section{DISCUSSION AND CONCLUSION}

In this paper, we have formulated the exploratory hypothesis testing and analysis problem and proposed a data mining 
approach to solve the problem. Conventional hypothesis testing allows just one or a few hypotheses to be tested at one time, while exploratory hypothesis testing enables researchers to use computational methods to examine large numbers of hypotheses and to identify those that have a reasonable chance of being true. In this fashion, human oversights and limitations can be complemented by computers.

This is our first attempt to connect hypothesis testing to database concepts and terminology by building on and extending existing data mining techniques. Our approach still has to be improved in many aspects:

- Controlling false positive rate. Controlling false positive rate in multiple hypothesis testing is a hard problem. This problem deserves much more discussion than what have been done in the paper. We used Bonferroni correction [1] and Benjamini and Hochberg's method [4] to control false positive rate. These two methods are very simple but they may inflate the number of false negatives unnecessarily. We will conduct an in-depth study of different correction methods in our system.

- Measures for hypothesis generation and analysis. We used the absolute difference between two sub-populations to measure the domain significance. Other measures can be used here as well. For example, odds ratio and relative risk have been commonly used when the target attribute is nominal. For hypothesis analysis, we have defined two simple measures, Contribution and DiffLift, to measure the impact of an item or an attribute to a hypothesis. We do not claim that the measures used in the paper are the best, but we believe that they do capture useful information and can serve the purpose. In association rule mining, many interestingness measures have been proposed, but none of them is superior to all the others in every aspect. The situation is the same here. It will be difficult to find a measure that is better than all other possible measures in every aspect. We can use several measures together to get a more comprehensive understanding of the data.

- Concise representations of hypotheses. It is well-known that the complete set of frequent patterns contains a lot of redundant information. Several concepts have been proposed to remove the redundancy from the complete set of frequent patterns, such as closed patterns, maximal patterns, generators, equivalent classes. The same thing can be done for hypotheses. The techniques developed for concise representations of frequent patterns can be employed and extended to represent hypotheses concisely.

- Visualization of the generated hypotheses. It is very important to organize and present the hypotheses properly so that users can explore them to find interesting things easily, especially when many significant hypotheses are generated. Currently, we rank the hypotheses in ascending order of their p-values and highlight the hypotheses with Simpson's Paradox. We will explore more sophisticated methods to visualize the output in the future.
It is not our intention to replace conventional hypothesis testing with exploratory hypothesis testing. Instead, we believe that the two approaches are complementary to each other. Exploratory hypothesis testing can be employed to find potentially interesting things in the data via extensive computation, which is tedious and time-consuming for scientists to do manually, especially with the large-scale datasets that are available nowadays. The generated significant hypotheses provide the starting points for scientists to examine further. To confirm these hypotheses, scientists still need to perform a rigorous evaluation using conventional hypothesis testing.

\section{ACKNOWLEDGMENT}

This work is supported in part by Singapore Agency for Science, Technology \& Research grant SERC 10210100030.

\section{REFERENCES}

[1] H. Abdi. Bonferroni and Šidák corrections for multiple comparisons". N.J. Salkind (ed.). Encyclopedia of Measurement and Statistics. Thousand Oaks, CA: Sage, 2007.

[2] R. Agrawal, T. Imielinski, and A. N. Swami. Mining association rules between sets of items in large databases. In SIGMOD, pages 207-216, 1993.

[3] S. D. Bay and M. J. Pazzani. Detecting change in categorical data: mining contrast sets. In SIGKDD, pages 302-306, 1999.

[4] Y. Benjamini and Y. Hochberg. Controlling the false discovery rate: a practical and powerful approach to multiple testing. Journal of the Royal Statistical Society, 57(1):125-133, 1995.

[5] G. Dong and J. Li. Efficient mining of emerging patterns: Discovering trends and differences. In SIGKDD, pages 43-52, 1999.

[6] A. A. Freitas. On objective measures of rule surprisingness. In $P K D D$ pages 1-9, 1998.

[7] L. Geng and H. J. Hamilton. Interestingness measures for data mining: a survey. ACM Computing Surveys, 38(3), 2006.

[8] B. Goethals and M. J. Zaki, editors. Proceedings of the ICDM 2003 Workshop on Frequent Itemset Mining Implementations, volume 90 of CEUR Workshop Proceedings, 2003.

[9] W. S. Gosset(Student). The probable error of a mean. Biometrika, 6(1):1-25, 1908.

[10] J. Han, J. Pei, and Y. Yin. Mining frequent patterns without candidate generation. In SIGMOD, pages 1-12, 2000.

[11] A. Kirsch, M. Mitzenmacher, A. Pietracaprina, G. Pucci, E. Upfal, and F. Vandin. An efficient rigorous approach for identifying statistically significant frequent itemsets. In PODS, pages 117-126, 2009.

[12] B. Liu, W. Hsu, L.-F. Mun, and H.-Y. Lee. Finding interesting patterns using user expectations. TKDE, 11(6):817-832, 1999.

[13] B. Liu, K. Zhao, J. Benkler, and W. Xiao. Rule interestingness analysis using olap operations. In SIGKDD, pages 297-306, 2006.

[14] G. Liu, J. Li, and L. Wong. A new concise representation of frequent itemsets using generators and a positive border. KAIS, 17(1):35-56, 2008.

[15] G. Liu, H. Lu, and J. X. Yu. Cfp-tree: A compact disk-based structure for storing and querying frequent itemsets. Information Systems, 32(2):295319, 2007.

[16] H. B. Mann and D. R. Whitney. On a test of whether one of two random variables is stochastically larger than the other. Annals of Mathematical Statistics, 18:50-60, 1947.

[17] H. J. Motulsky. http://www.graphpad.com/www/book/choose.htm.

[18] A. Silberschatz and A. Tuzhilin. On subjective measures of interestingness in knowledge discovery. In SIGKDD, pages 275-281, 1995.

[19] E. H. Simpson. The interpretation of interaction in contingency tables. Journal of the Royal Statistical Society, 13(2):238-241, 1951.

[20] P.-N. Tan, V. Kumar, and J. Srivastava. Selecting the right interestingness measure for association patterns. In $S I G K D D$, pages 32-41, 2002.

[21] F. Yates. Contingency table involving small numbers and the $\chi^{2}$ test. Supplement to the Journal of the Royal Statistical Society, 1(2):217-235, 1934.

[22] L. Zhang, B. Liu, J. Benkler, and C. Zhou. Finding actionable knowledge via automated comparison. In ICDE, pages 1419-1430, 2009. 